Kompass

Pneumologie

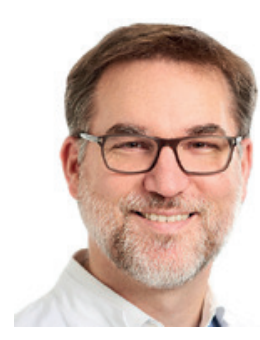

\section{Lars Hagmeyer}

Krankenhaus Bethanien Solingen, Institut für Pneumologie an der Universität zu Köln, Solingen, Deutschland

Kompass Pneumol 2021;9:1-2

DOI: $10.1159 / 000514473$

\title{
COVID-19: Zwischenbilanz nach einem Jahr
}

Das Coronavirus SARS-CoV-2 (severe acute respiratory syndrome coronavirus-2) beherrscht weiterhin die Schlagzeilen in Deutschland, ja auf der ganzen Welt. In radikaler Weise hat die Pandemie das gesellschaftliche Leben und den medizinischen Alltag verändert.

Die akribische Auswertung verfügbarer Daten aus der Versorgungsmedizin und intensive Forschungsaktivitäten ermöglichten innerhalb kürzester Zeit einen erheblichen Erkenntniszugewinn. Dieser hat unmittelbare Auswirkungen auf die tägliche Arbeit in Klinik und Praxis. Die aktuelle Ausgabe des Kompass Pneumologie versucht eine Standortbestimmung und trägt jüngste medizinische Erkenntnisse zusammen, die in den letzten Monaten gewonnen wurden. Die hier getroffene Auswahl kann nur einen Bruchteil der klinischen Studien abbilden, die für die praktische Pneumologie relevant sind.

Nur ein Teil der SARS-CoV-2-Infizierten entwickelt Symptome und erkrankt an COVID-19 (Corona Virus Disease 2019). Bei Erstpräsentation der Patienten ist eine Abschätzung des Risikos für einen schweren Verlauf von hoher Bedeutung. Indikatoren, die eine wahrscheinlich erforderliche intensivmedizinische Behandlung, prognostisch relevante Komplikationen oder das Sterberisiko vorauszusagen helfen, sind hier von besonderem Interesse.
Die COVID-19-Pneumonie und die Komplikation des schweren akuten Lungenversagens mit dem Bild eines ARDS stellen in der Pandemie die häufigsten und prognostisch relevantesten Herausforderungen für den Pneumologen dar. Es kann jedoch auch zu anderen Krankheitsmanifestationen kommen, die den pneumologischen Fachbereich unmittelbar betreffen. Die Übersichtsarbeit von Samhati Mondal et al. beleuchtet die Risikofaktoren und die Pathophysiologie thromboembolischer Erkrankungen mit Fokus auf die COVID-19-Erkrankung. Die jüngst gemeinsam veröffentlichten Empfehlungen der amerikanischen und europäischen Pneumologengesellschaften (American Thoracic Society, ATS; European Respiratory Society, ERS) zur Behandlung von COVID-19 fasst Johannes Knoch in seinem Wissenstransfer zusammen. Der Artikel führt eindrücklich vor Augen, wie wichtig die kritische Würdigung der verfügbaren Evidenz bei der Erstellung von Leitlinien und Empfehlungen ist. So wird in dem Dokument zu den Therapieansätzen mit Dexamethason, Remdesivir, Hydroxychloroquin und Antikoagulanzien dezidiert Stellung genommen.

Stefanie Keymel stellt in Ihrem Transfer in die Praxis die Arbeit von Ahmed M. Abdalhadi und Kollegen vor, die eine Kasuistik mit beidseitigen exsudativen lymphozytären Pleuraergüssen bei COVID-19 erläutern. 
Helmut Frohnhofen zeigt mit der Arbeit von Ting Guo und Mitarbeitern die klinischen Besonderheiten der COVID-19-Erkrankung bei älteren Menschen auf. So kann gerade bei den besonders alten Patienten trotz symptomarmem Erkrankungsbeginn ein komplikationsträchtiger und letztlich vital bedrohlicher Verlauf eintreten. Auch für dieses Patientenkollektiv wurden erste klinische Risikoindikatoren identifiziert. Die COVID-19-Therapie kann beim geriatrischen Patienten nicht unreflektiert vereinheitlicht werden, sondern sollte vielmehr nach sorgfältiger individueller Abwägung gesteuert werden.

Stefan Krüger schließlich diskutiert in seinem Wissenstransfer die Studie von Hannah Burke und Kollegen, die darin ein breites Zytokinpanel bei stationär aufge- nommenen COVID-19-Patienten untersucht haben. Die Arbeit zeigt, dass Zytokin-Analysen ein weiteres Instrument zur Prognoseabschätzung darstellen könnten. Andererseits zeigt sich die Dynamik des vieldiskutierten «Zytokinsturms» heterogen und komplex, so dass sich noch kein solitärer Zytokin-Biomarker abzeichnet, der Einzug in die klinische Routine halten könnte.

In der Rubrik «Erfahrung aus der Praxis» anaIysieren Massa Zantah und Mitarbeiter in Ihrem Patientenkollektiv retrospektiv die seltene aber klinisch hochrelevante Komplikation eines Pneumothorax bei COVID-19. Besonders bei beatmeten Patienten und radiomorphologisch auffälligen Milchglasveränderungen und Konsolidierungen sollte bei unklarer klinischer Verschlechterung ein Pneumothorax ausgeschlossen werden.

Insgesamt zeigt sich auch mit dieser Auswahl an Arbeiten, dass die Pneumologie ein facettenreiches Fach ist und in besonderem Maße bei der Bewältigung der COVID19-Pandemie gefordert ist. Im historischen Vergleich zeichnet sich die derzeitige Pandemie dadurch aus, dass reger internationaler Erfahrungsaustausch und intensive Forschung möglich sind und diese Früchte tragen.

Ihr

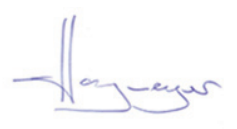

Dr. Lars Hagmeyer 